\title{
RursuSpicae
}

Transmission, réception et réécriture de textes, de I'Antiquité au Moyen Âge

$2 \mid 2019$

Le Physiologus. Manuscrits anciens et tradition médiévale

\section{Panorama des manuscrits et nouvelles ressources pour l'étude de la tradition manuscrite du Physiologus latin}

Overview of the Manuscripts and New Resources for the Study of the Manucript Tradition of the Latin Physiologus

\section{Emmanuelle Kuhry}

\section{(2) OpenEdition}

Journals

\section{Édition électronique}

URL : http://journals.openedition.org/rursuspicae/924

DOI : $10.4000 /$ rursuspicae. 924

ISSN : 2557-8839

Éditeur :

Université Nice-Sophia Antipolis, IRHT - Institut de recherche et d'histoire des textes

\section{Référence électronique}

Emmanuelle Kuhry, «Panorama des manuscrits et nouvelles ressources pour l'étude de la tradition manuscrite du Physiologus latin », RursuSpicae [En ligne], 2 | 2019, mis en ligne le 20 décembre 2019, consulté le 29 mars 2021. URL : http://journals.openedition.org/rursuspicae/924 ; DOI : https:// doi.org/10.4000/rursuspicae.924

Ce document a été généré automatiquement le 29 mars 2021.

RursuSpicae 


\title{
Panorama des manuscrits et nouvelles ressources pour l'étude de la tradition manuscrite du Physiologus latin
}

Overview of the Manuscripts and New Resources for the Study of the Manucript Tradition of the Latin Physiologus

\author{
Emmanuelle Kuhry
}

\section{Avant-propos}

1 Dans cette contribution, j'entends mettre à disposition trois ressources pour l'étude du Physiologus latin : une liste des manuscrits du Physiologus latin classés par version, dont le total atteint 153 entrées; un document présentant les tables des matières comparées des principaux manuscrits dans toutes les versions; la liste des codes utilisés dans les tables des chapitres.

2 Ces ressources sont précédées d'une rapide introduction, qui précise le cadre dans lequel les ressources sont établies. Elle vise à donner les grandes lignes de la tradition manuscrite du Physiologus latin, en passant en revue les versions anciennes telles qu'elles ont été identifiées par la critique, puis les versions du bestiaire qui naît de la version $\mathrm{B}$ et se développe en différentes familles entre le $\mathrm{X}^{\mathrm{e}}$ et le XIII ${ }^{\mathrm{e}}$ siècle.

$3 \mathrm{Au}$ carrefour des traditions zoologiques et exégétique égyptienne, grecque, juive et chrétienne, le texte du Physiologus transmis à l'Occident latin connaît un succès extraordinaire tout au long du Moyen-Âge, qui est attesté par les nombreux remaniements qu'il subit : réorganisations, enrichissements, abréviations, traductions vers les langues vernaculaires, versifications. Toutes versions latines confondues, le texte du Physiologus latin et de son héritier médiéval le bestiaire sont transmis dans 
plus de deux-cents manuscrits connus, un nombre certainement encore bien inférieur à la réalité.

4 Ce travail est issu d'un post-doctorat réalisé au CEPAM de Nice sous la direction d'A. Zucker.

\section{La tradition manuscrite du Physiologus}

5 Le Physiologos est un bestiaire moralisé écrit en grec dans un milieu alexandrin du $\mathrm{II}^{\mathrm{e}}$ ou du III siècle après J.- $\mathrm{C}^{1}$. Le texte de la première collection grecque, comptant quarantehuit animaux, pierres et arbres, est traduit en latin, probablement avant 500, et en tout cas avant le milieu du VIII ${ }^{\mathrm{e}}$ siècle ${ }^{2}$. D'autres traductions sont diffusées : éthiopienne, copte, syriaque, arabe, arménienne et géorgienne ${ }^{3}$.

\section{Les versions anciennes}

6 Au sein des versions latines, qui vont nous occuper dans cette contribution, la version Y, nommée d'après l'édition de F. J. Carmody (CARMODY, 1941), est considérée comme issue de la traduction vers le latin d'un original grec proche du manuscrit $\Pi$ dont elle reprend l'ordre des chapitres. Elle est livrée, dans des états divers, dans six témoins dont le plus ancien date de la première moitié du VIII ${ }^{e}$ siècle mais contient également des chapitres de version $B$.

7 La version C a été éditée aux côtés des versions A et B par C. Cahier et A. Martin (CAHIER ET MARTIN, 1851). Livrant comme la version $Y$ des translittérations de termes grecs, elle est supposée issue de la traduction d'une autre version grecque (famille $\Sigma$ as de la première rédaction). Elle est transmise dans deux témoins des $\mathrm{IX}^{\mathrm{e}}$ et $\mathrm{X}^{\mathrm{e}}$ siècles.

8 L'origine de la version B n'est pas élucidée. F. Sbordone (SBORDONE, 1949) a émis l'hypothèse que le Physiologos grec a connu deux traductions vers le latin : $x$ (que nous connaissons sous « $C »$ ) et $y$, dont l'état originel ne nous est pas parvenu mais qui aurait donné naissance aux versions Y ( $a$ chez Sbordone) et B ( $b$ chez Sbordone). La version B en tant que telle n'est transmise que dans trois manuscrits des $\mathrm{IX}^{\mathrm{e}}$ et $\mathrm{X}^{\mathrm{e}}$ siècles. Elle est éditée de manière imparfaite par F. J. Carmody (CARMODY, 1939).

9 Enfin la version A s'apparente plutôt à une compilation de chapitres B et de chapitres $Y$, dans un ordre qui lui est propre. On ne considère en général qu'un seul témoin $d u X^{e}$ siècle pour cet état de texte, mais deux autres manuscrits peuvent lui être rattachés, l'un du XI ${ }^{e}$ siècle, l'autre du XII ${ }^{e}$-XIII ${ }^{e}$ siècle.

\section{Le bestiaire}

10 La version $B$ est celle qui connaît le plus de succès au Moyen Âge ${ }^{4}$. Vers le $X^{e}$ siècle, le Physiologus B reçoit des additions constituées de notices provenant des Étymologies d'Isidore de Séville, ajoutées à la fin de chaque chapitre, et donne ainsi naissance à la version B-Is. Un remaniement de cette version aboutit à la version H-B-Is au XII ${ }^{\mathrm{e}}$ siècle (BAXTER, 1998 : 83-105).

11 De ces deux états de texte dépendent le bestiaire de Seconde Famille (CLARK, 2006), le bestiaire de version $\mathrm{H}$, édité comme second livre du recueil factice De bestiis et aliis rebus attribué à tort à Hugues de Saint-Victor ${ }^{5}$, et le bestiaire dit « de Transition », supposé 
par F. McCulloch se placer entre B-Is et la Seconde Famille, mais qui emprunte en réalité à ces deux familles (DINES, 2012). Enfin le bestiaire de Troisième famille (XIII ${ }^{\mathrm{e}}$ siècle) tire son contenu principalement de la Seconde Famille et des Étymologies d'Isidore de Séville (wHITE, 2009). Quant à la quatrième famille identifiée par M. R. James, elle sort du cadre du Physiologus de par sa dépendance principale envers le De proprietatibus rerum de Barthélémy l'Anglais.

Il faut mentionner également les Dicta Chrysostomi, qui constituent une version à part entière, indépendante des bestiaires issus de $B$, mais dont est tirée une partie du contenu de la version $\mathrm{H}$, et dont la tradition est très riche. $\mathrm{F}$. Sbordone en a supposé une origine en France dès le IX $\mathrm{I}^{\mathrm{e}}$ siècle et selon lui elle se divise en six familles (SBORDONE, 1949 : 253-72). Il s'agit de la première version à séparer bestiae et oiseaux, avant la version H-B-Is au XII ${ }^{e}$ siècle. Elle a reçu plusieurs éditions anciennes dont une édition critique (WILHELM, 1914).

Le Physiologus Theobaldi constitue un autre remaniement $d u \mathrm{XI}^{\mathrm{e}}$ ou du début du $\mathrm{XII}^{\mathrm{e}}$ siècle. Il serait originaire de France ou d'Italie et est transmis dans plus de soixante-dix manuscrits identifiés, ce qui en fait la version du Physiologus possédant la tradition la plus riche (EDEN, 1972).

\section{Objectif du présent travail et avertissement}

On trouvera en annexe de cette publication un ensemble de trois ressources pour l'étude du Physiologus latin:

\section{Une liste des manuscrits du Physiologus latin et des bestiaires dérivés}

Cette liste, comprenant actuellement 153 manuscrits, est le produit d'une compilation d'un certain nombre de travaux que l'on trouvera dans la bibliographie ci-après ${ }^{6}$.

Cette liste ne vise ni à l'exhaustivité, ni à la complétude des informations. En particulier dans le cas de la Seconde Famille et des Dicta Chrysostomi, il est certain que de nouvelles études permettront de mettre au jour des témoins jusque là inconnus.

Sont donnés pour chaque manuscrit les éléments d'identification (ville, bibliothèque, cote), la date présumée de copie, dans la mesure du possible la foliotation correspondant au Physiologus ou au bestiaire.

18 Cette liste se veut donc une porte d'entrée vers la tradition manuscrite du Physiologus latin. Ainsi on trouvera, lorsque c'est possible, des liens vers la reproduction et vers une description en ligne.

Enfin certaines entrées débutent par un sigle précédé d'un astérisque : il s'agit du sigle utilisé dans la table des chapitres (ressource suivante).

Toutes les adresses et liens hypertexte ont été consultés en décembre 2019 ; il se peut que certains des liens hypertexte s'invalident dans un avenir plus ou moins lointain.

Le Physiologus Theobaldi n'a pas été pris en compte dans ce recensement. Sur cette œuvre, on se reportera à l'édition de référence de P. T. Eden citée plus haut. 


\section{Les tables des matières comparées des principaux manuscrits dans toutes les versions}

L'objectif de cette table est de permettre le repérage rapide du passage recherché au sein d'un manuscrit, de fournir un premier moyen d'identification de l'état du texte présent dans un manuscrit, et de faciliter les comparaisons entre versions et entre manuscrits d'une même version.

Tous les témoins des versions anciennes ont été considérés. En ce qui concerne les bestiaires issus de la version B et les Dicta Chrysostomi, une sélection a été opérée, en laissant une place aux manuscrits atypiques au sein de leur version.

Les manuscrits sont désignés sous la forme d'un sigle que l'on retrouvera avec un astérisque au début de chaque entrée dans la liste de témoins. Les sigles suivis de « (T) » indiquent le contenu de la table des matières figurant dans le volume manuscrit (à comparer avec la table des contenus réels).

La table des manuscrits grecs $G$ et $A$, ainsi que celle du manuscrit grec $\Pi$, ont été ajoutées à des fins de comparaison.

Le fichier a été mis en page en format A3, afin de permettre de représenter toutes les tables côte à côte. La ressource est proposée dans une version en noir et blanc ou une version en couleurs, au sein de laquelle les couleurs désignent les parties du règne naturel :

- rouge saumon pour les bestiae

- bleu pour les poissons, cétacés et crustacés

- vert pour les reptiles et batraciens

- fuschia pour les insectes, vers

- jaune pour les oiseaux

- marron pour les plantes

- gris pour les pierres

- vert kaki pour les monstres, uniquement dans la Troisième famille

- blanc pour les personnages humains (Amos et Adam)

\section{Une liste des sigles utilisés dans les tables pour représenter les zoonymes, noms de pierres et de plantes}

Cette liste est complémentaire de la table. Elle fait correspondre un code à trois lettres aux zoonymes latin et français. Elle a été réalisée en collaboration avec A. Zucker.

\section{Sigles utilisés pour désigner les versions}

Dans cette publication, nous utilisons, pour désigner les témoins, des sigles correspondant à chaque version :

\begin{tabular}{|l|l|}
\hline Version & Sigle \\
\hline$Y$ & $Y$ \\
\hline
\end{tabular}




\begin{tabular}{|l|l|}
\hline C & C \\
\hline B & B \\
\hline A & A \\
\hline B-Is & B-Is \\
\hline Seconde Famille & B-Bes \\
\hline H & B-Hu \\
\hline « Transition » & B-Tr \\
\hline Troisième famille & B-Tert \\
\hline Dicta Chrysostomi & DC \\
\hline
\end{tabular}

\section{Conclusion}

Le chercheur trouvera publiés ici de manière inédite trois outils pour l'étude du Physiologus latin et des bestiaires à partir des manuscrits. Il s'agit en effet de la première mise en commun d'informations sinon éparses dans la littérature. Ces trois ressources sont les premières de leur genre à prendre en compte l'ensemble des versions du texte. En ce qui concerne la liste des manuscrits, la synthèse de $\mathrm{F}$. McCulloch en 1962 était la dernière tentative d'embrasser la totalité de la tradition manuscrite. Une mise à jour était donc grandement nécessaire. La liste qu'on trouvera ici rassemble les informations disponibles dans les principales travaux dédiés au Physiologus latin et aux bestiaires, et ajoute plusieurs témoins significatifs. La question des tables des chapitres est abordée dans l'une ou l'autre publication (par exemple: BAXTER, 1998 et KAY, 2016), souvent dans une perspective comparatiste comme ici, mais jamais de manière générale.

Les principales avancées récentes dans la connaissance de la tradition manuscrite du Physiologus latin, qui ne peuvent malheureusement être abordées ici dans toute leur complexité, seront détaillées dans un article à paraître en collaboration avec A. Zucker.

\section{BIBLIOGRAPHIE}




\section{Éditions de textes}

CAHIER Charles, et Arthur MARTIN (éd.), Mélanges d'archéologie, d'histoire et de littérature, 2-4, Paris, 1851.

CARMODY Francis J. (éd.), Physiologus Latinus Versio Y, Berkeley, 1941.

CARMODY Francis J. (éd.), Physiologus latinus : éditions préliminaires, versio B, Paris, 1939.

CLARK Willene B. (éd.), A Medieval Book of Beasts: the Second-Family Bestiary ; Commentary, Art, Text and Translation, Woodbridge, 2006.

WHITE Cynthia (éd.), From the Ark to the Pulpit: an Edition and Translation of the "Transitional » Northumberland Bestiary (13th century), Louvain-la-Neuve, 2009.

WILHELm Friedrich (éd.), Denkmäler deutscher Prosa des 11. und 12. Jahrhunderts, München, 1914, (Münchener Texte, 8).

ZUCKER Arnaud (trad.), Physiologos : le bestiaire des bestiaires, Grenoble, 2004.

\section{Travaux}

BAXTER Ron, Bestiaries and their users in the Middle Ages, Sutton, 1998.

CLARK Willene B., "Four Latin bestiaries and De bestiis et aliis rebus ", in Bestiaires médiévaux. Nouvelles perspectives sur les manuscrits et les traditions textuelles, VAN DEN ABEELE, B., (éd.), Louvainla-Neuve, 2005, p. 49-69.

HENKEL Nikolaus, Studien zum Physiologus im Mittelalter, Tübingen, 1976.

JAMES Montague R., The Bestiary, Being a Reproduction in full of the Ms. Ii.4.26 in the University Library, Oxford, 1928.

KAY Sarah, « The English Bestiary, the Continental Physiologus, and the Intersections between Them », Medium Aevum, 85 (1), 2016, p. 118-42.

KUHRY Emmanuelle, « Dictionnaires, distinctions, recueils de propriétés en milieu cistercien : outils pour la prédication, sources pour l'étude de la nature ", in Les Cisterciens et la transmission des textes (XII ${ }^{e}$-XVIII ${ }^{e}$ siècles), TURCAN-VERKERK, A.-M., FALMAGNE, Th., STUTZMANN, D., et GANDIL, P. (éd.), Turnhout, 2018, (Bibliothèque d'histoire culturelle du Moyen Âge, 18), p. 285-337.

KUHRY Emmanuelle, «Les chapitres sur les poissons et créatures aquatiques dans la tradition manuscrite du Physiologus latinus et des bestiaires latins et leur réception dans quelques encyclopédies médiévales ", in Inter litteras et scientias. Recueil d'études en hommage à Catherine Jacquemard, AVENEL, M.-A. - GAUVIN, B., (éd.), Caen, 2019, p. 117-50.

LAZARIS Stavros, Le Physiologus grec, I : la réécriture de l'histoire naturelle antique, Sismel, 2016. MCCULLoch Florence, Mediaeval Latin and French bestiaries, Chapel Hill, 1962.

ORLANDI Giovanni, « La tradizione del Physiologus e i prodromi nel bestiaro latino », in L'uomo di fronte al mondo animale nell'alto Medioevo, Spolète, 1985, p. 1057-1106.

PAKIS Valentine A., «Contextual Duplicity and Textual Variation: The Siren and Onocentaur in the Physiologus Tradition », Mediaevistik, 23, 2010, p. 115-85.

SBORDONE Francesco, Ricerche sulle fonti e sulla composizione del Physiologus greco, Napoli, 1936. 
SBORDONE Francesco, « La tradizione manoscritta del Physiologus latino », Athenaeum, 27, 1949, p. 246-80.

STEWART Patricia, The medieval bestiary and its textual tradition, Thèse de l'University of St. Andrews, 2012.

\section{ANNEXES}

\section{Annexe 1 : liste des manuscrits du Physiologus latin et des bestiaires dérivés}

\section{Version $Y$}

['Y3] Bern, Burgerbibliothek, 611, s. 8-9, f. 116v-138v

repr. : http://www.e-codices.unifr.ch/fr/bbb/0611/116v/0/Sequence-1570

[ $\left.{ }^{*} Y 2\right]$ München, Bayerische Staatsbibliothek, Clm 14388, s. 9-10, f. 172v-183v

repr. : http://daten.digitale-sammlungen.de/ db/0002/bsb00022465/images/

index.html?fip=193.174.98.30\&seite=350\&pdfseitex

desc. : https://opacplus.bsb-muenchen.de/title/BV021491063

[Y1] München, Bayerische Staatsbibliothek, Clm 19417, s. 9, f. 28v-70v

repr. : http://daten.digitale-sammlungen.de/ db/0003/bsb00036883/images/

index.html?

id=00036883\&groesser $=\&$ fip $=$ eayafsdrewqyztseayafsdrwxdsydfsdr\&no $=40 \&$ seite $=62$

desc. : https://opacplus.bsb-muenchen.de/title/BV035383883

$\left.{ }^{*}{ }^{*} 5\right]$ Paris, BNF, NAL 455, s. 10, f. 3-8v

repr. : https://gallica.bnf.fr/ark:/12148/btv1b52507114b

desc. : https://archivesetmanuscrits.bnf.fr/ark:/12148/cc71179s, seul le premier cahier subsiste

[*Y6] Saint-Gall, Stiftsbibliothek, 230, s. 9, f. 510-518

repr. : http://www.e-codices.unifr.ch/de/csg/0230/510/0/Sequence-415

[*Y4] Wolfenbüttel, Herzog August Bibliothek, Cod. Guelf. 131 Gud. lat. (4435), s. 11, f. $159-168$

desc. : http://diglib.hab.de/?db=mss\&list=ms\&id=131-gud-lat\&catalog=Milchsack

\section{Version C}

[ $\left.{ }^{*} C 1\right]$ Bern, Burgerbibliothek, 318, s. 9, f. 7-22v

repr. : http://www.e-codices.unifr.ch/de/bbb/0318/7r/0/Sequence-34

$\left[{ }^{*} C 2\right]$ Wolfenbüttel, Herzog August Bibliothek, Cod. Guelf. 148 Gud. lat. (4452), s. 10, f. $98 \mathrm{v}$...

repr. : http://diglib.hab.de/?db=mss\&list=ms\&id=148-gud-lat\&lang=en

desc. : http://diglib.hab.de/?db=mss\&list=ms\&id=148-gud-lat\&catalog=Milchsack 


\section{Version B}

[ $\left.{ }^{*} B 1\right]$ Bern, Burgerbibliothek, 233, s. 9 (inc.), f. 1-13

desc. : http://katalog.burgerbib.ch/detail.aspx?ID=130292

['B3] Montecassino, Archivio dell'Abbazia, 323, s. 9, f. p. 65-110

[*B2] Oxford, Bodl. Lib., Auct. T.2.23, s. 10, f. 127-155

desc. : https://medieval.bodleian.ox.ac.uk/catalog/manuscript_774

Hamburg, Staats-und Universitätsbibliothek, 47 in scrinio, s. 14, f. 65r-69v, B/Y/

Seconde Famille

repr. : https://resolver.sub.uni-hamburg.de/kitodo/HANSh782

desc. : http://bilder.manuscripta-mediaevalia.de/hs//CIMA/CIMA\%2048.pdf, incomplet

\section{Version « $A$ »}

[ $\left.{ }^{*} A 1\right]$ Bruxelles, KBR, 10066-77, s. 10, f. 140v-156v

repr. : https://opac.kbr.be/Library/doc/SYRACUSE/17347006

[ ${ }^{*}$ A3] Paris, BNF, lat. 2825, s. 11 (ex.), f. 49-56, A ?

repr. : https://gallica.bnf.fr/ark:/12148/btv1b105420770.image

desc. : https://archivesetmanuscrits.bnf.fr/ark:/12148/cc60613q

[*A2] Rouen, BM, 638, s. 12-13, f. 232-242, A ?

desc. : https://ccfr.bnf.fr/portailccfr/ark:/06871/004D08A11587

\section{Version B-Is}

Bruxelles, KBR, 8536-43, s. 13, f. 98-116

Bruxelles, KBR, X 106, s. 13

[ $\left.{ }^{*} B-I s 1\right]$ Città del Vaticano, BAV, Pal. lat. 1074, s. 10-11, f. 1-22

repr. : http://digi.ub.uni-heidelberg.de/diglit/bav_pal_lat_1074/0005?

sid=e4102049de6c90af9fa92e2991752e1a,

desc. : https://digi.ub.uni-heidelberg.de/diglit/walz1999/0323/image

London, BL, Cotton Vitellius D.I, s. 13, f. 1-29v, endommagé

[*B-Is7] London, BL, Royal 2 C XII, s. 13 (inc.), f. 133-145

Los Angeles, Getty Museum, Ludwig XV 3, s. 13, f. 73-116

repr. partielle: http://www.getty.edu/art/collection/objects/1436/unknown-hugo-offouilloy-bestiary-franco-flemish-about-1270/, olim London, Sion College, L 40.2 / L 28

Los Angeles, Getty Museum, Ludwig XV 4, s. 13, f. 67-102v

repr. partielle: http://www.getty.edu/art/collection/objects/1437/unknown-hugo-offouilloy-and-william-of-conches-de-natura-avium-de-pastoribus-et-ovibus-bestiariummirabilia-mundi-philosophia-mundi-on-the-soul-franco-flemish-fourth-quarterof-13th-century-after-1277/?dz=0.5000, 0.5119, 0.85, olim Dyson Perrins 26

Oxford, Bodl. Lib., Bodl. 602, s. 13, f. 1-36

repr. partielle: https://digital.bodleian.ox.ac.uk/inquire/p/b2e51d2c-6464-453d-b670- 
c51960808105

desc. : https://medieval.bodleian.ox.ac.uk/catalog/manuscript_1614

Oxford, Bodl. Lib., Douce 167, s. 13 (inc.), f. 1-12

repr. partielle: https://digital.bodleian.ox.ac.uk/inquire/p/

dffb4ecd-845b-4e24-9b25-470e4e599c08

desc. : https://medieval.bodleian.ox.ac.uk/catalog/manuscript_4541

Oxford, Bodl. Lib., Laud Misc. 247, s. 12 (inc.), f. 139v-166v

repr. partielle: https://digital.bodleian.ox.ac.uk/inquire/p/9a086667-538f-41da-aa47a424dc1cbeff

desc. : https://medieval.bodleian.ox.ac.uk/catalog/manuscript_7059

['B-Is14] Paris, BNF, NAL 873, s. 12, f. 37v-57

repr. : https://gallica.bnf.fr/ark:/12148/btv1b10548488f/f84.item.zoom

desc. : https://archivesetmanuscrits.bnf.fr/ark:/12148/cc715425

Tours, BM, 312, s. 15, f. 303-326v

repr. partielle: https://bvmm.irht.cnrs.fr/consult/consult.php?reproductionId $=8418$

desc. : https://ccfr.bnf.fr/portailccfr/ark:/06871/004D37A12943

\section{Sous-version H-B-Is}

[ $\left.{ }^{*} B-I s 4\right]$ Cambridge, Corpus Christi College, 22, s. 12, f. 162-169

repr. : https://parker.stanford.edu/parker/catalog/xd956wx5361, premier f. manquant, Cambridge, UL, Mm.6.15, s. 14, f. 186v-197, incomplet

[ $\left.{ }^{*} B-I s 3\right]$ London, BL, Stowe 1067, s. 12 (inc.), f. 1-15v

repr. : http://www.bl.uk/manuscripts/Viewer.aspx?ref=stowe_ms_1067_fs001r

desc. : http://www.bl.uk/manuscripts/FullDisplay.aspx?ref=Stowe_MS_1067

New Haven, Yale UL, Beinecke 851, s. 13, f. 8-19v

repr. : https://brbl-dl.library.yale.edu/vufind/Record/3586828

\section{Seconde Famille}

['B-Bes6] Aberdeen, UL, 24, s. 12 (ex.), f. 1-103

repr. : https://www.abdn.ac.uk/bestiary/

Augsburg, UB, Cod.II.1.2.109, s. 15, f. 128v-153v, incomplet

Bruxelles, KBR, 8340, s. 14, f. 183-215

Cambridge, Corpus Christi College, 53, s. 14 (inc.), f. 189-210

repr. : https://parker.stanford.edu/parker/catalog/gs233db8425

Cambridge, Fitzwilliam Museum, 379, s. 14 (inc.), f. 9-30v

Cambridge, Gonville et Caius Coll., 109, s. 13, f. 110-133

Cambridge, Gonville et Caius Coll., 372, s. 13, f. 1-64

Cambridge, Saint John's College, A.15, s. 13, f. 134v-143v

desc. :https://www.joh.cam.ac.uk/library/special_collections/manuscripts/ medieval_manuscripts/medman/A_15.htm, incomplet 
Cambridge, Saint John's College, C.12, s. 13, f. 11-23v

desc. : https://www.joh.cam.ac.uk/library/special_collections/manuscripts/ medieval_manuscripts/medman/C_12.htm, incomplet

Cambridge, UL, Dd.12.50, s. 13, incomplet

Cambridge, UL, Ii.4.26, s. 12, f. 1-74

repr. : https://cudl.lib.cam.ac.uk/view/MS-II-00004-00026/11

Canterbury, Cathedral Library, Lit. D 10, s. 13-14

Città del Vaticano, BAV, Vat. lat. 2770, s. 13, f. 37-70v

desc. : https://digi.vatlib.it/view/MSS_Vat.lat.2770

Copenhagen, KB, Gl. kgl. 1633 4o, s. 14, f. 1-76v

repr. : http://www.kb.dk/permalink/2006/manus/221/eng/1+recto/?var

Den Haag, Museum Mermanno, 10 B 25, s. 15

Douai, BM, 711, s. 13 (ex.), f. 1-60v

repr. partielle: https://bvmm.irht.cnrs.fr/resultRecherche/resultRecherche.php? COMPOSITION_ID=5981

desc. : https://ccfr.bnf.fr/portailccfr/ark:/06871/004D06A12912

Durham, UL, Cosin V.II.5, s. 14, f. 101-132

desc. : https://www.dur.ac.uk/library/asc/theme/medmss/apvii5/

Le Mans, BM, 84, s. 13, f. 3-72

repr. : https://bvmm.irht.cnrs.fr//consult/consult.php?reproductionId=5462 desc. : https://ccfr.bnf.fr/portailccfr/ark:/06871/004D25010683

[*B-Bes1] London, BL, Add. 11283, s. 12 (inc.), f. 1-41

repr. : http://www.bl.uk/manuscripts/Viewer.aspx?ref=add_ms_11283_fs001r

desc. : http://www.bl.uk/manuscripts/FullDisplay.aspx?ref=Add_MS_11283

London, BL, Burney 327, s. 13 (inc.)

desc. : https://www.bl.uk/catalogues/illuminatedmanuscripts/record.asp?

MSID $=1515 \&$ ColliD $=18 \& N S t a r t=327$

[*B-Bes15] London, BL, Harley 3244, s. 13 (inc.), f. 36-71v

repr. : http://www.bl.uk/manuscripts/Viewer.aspx?ref=harley_ms_3244_f036r

desc. : http://www.bl.uk/manuscripts/FullDisplay.aspx?ref=Harley_MS_3244

London, BL, Harley 4751, s. 12 (ex.), f. 1-74v

repr. : http://www.bl.uk/manuscripts/Viewer.aspx?ref=harley_ms_4751_fs001r

desc. : http://www.bl.uk/manuscripts/FullDisplay.aspx?ref=Harley_MS_4751

[*B-Bes7] London, BL, Royal 12 F XIII, s. 12-13, f. 1-141

repr. : http://www.bl.uk/manuscripts/Viewer.aspx?ref=royal_ms_12_f_xiii_fo03r

desc. : http://www.bl.uk/manuscripts/FullDisplay.aspx?ref=Royal_MS_12_F_XIII

London, BL, Sloane 3544, s. 13, f. 1-44

desc. : https://www.bl.uk/catalogues/illuminatedmanuscripts/record.asp?

MSID $=6510 \&$ CollID $=9 \&$ NStart $=3544$ 
New York, Morgan Library, 890, s. 14, f. 1-18

repr. : https://www.themorgan.org/collection/bestiary/159535/thumbs

desc. : https://www.themorgan.org/manuscript/159535

Nîmes, BM, 82, s. 16 (inc.), f. 1-90v

repr. : https://bvmm.irht.cnrs.fr/consult/consult.php?reproductionId=5332

desc. : https://ccfr.bnf.fr/portailccfr/ark:/06871/004D07020319

Oxford, Bodl. Lib., Ashmole 1511, s. 12 (ex.), f. 1-104

repr. : https://digital.bodleian.ox.ac.uk/inquire/p/

fb43631c-1f61-4865-806c-9fe59b5753ff

desc. : https://medieval.bodleian.ox.ac.uk/catalog/manuscript_290

Oxford, Bodl. Lib., Bodl. 533, s. 13, f. 1-29v

repr. partielle: https://digital.bodleian.ox.ac.uk/inquire/p/bffef55e-f424-41ba-

a34e-6f7662e9073c

desc. : https://medieval.bodleian.ox.ac.uk/catalog/manuscript_1556

Oxford, Bodl. Lib., Bodl. 764, s. 12 (ex.), f. 1-137

repr. : https://digital.bodleian.ox.ac.uk/inquire/p/6fde525f-c745-4969-

b203-1710bdfeb761

desc. : https://medieval.bodleian.ox.ac.uk/catalog/manuscript_1750

Oxford, Bodl. Lib., Bodl. 91, s. 14, f. 79-94v,

desc. : https://medieval.bodleian.ox.ac.uk/catalog/manuscript_1877

Oxford, Bodl. Lib., Douce 151, s. 14, f. 1-90

repr. partielle: https://digital.bodleian.ox.ac.uk/inquire/p/00e9ebb1-2bb4-4e61-bob0cd0de6d96978

desc. : https://medieval.bodleian.ox.ac.uk/catalog/manuscript_4529, copie de Ashmole 1511

Oxford, Bodl. Lib., Douce 88 A, s. 13 (ex.), f. 5-29

Oxford, Bodl. Lib., Rawlinson C77, s. 13, f. 1-3v

desc. : https://medieval.bodleian.ox.ac.uk/catalog/manuscript_8341, extraits

Oxford, Bodl. Lib., Tanner 110, s. 13, f. 1-7v

desc. : https://medieval.bodleian.ox.ac.uk/catalog/manuscript_8968, extraits ; Clark précise une affiliation à la Seconde Famille tandis que le catalogue indique que le bestiaire est en français.

Oxford, Saint John's College, 61, s. 13, f. 1-103

Oxford, Saint John's College, 178, s. 13 (ex.), f. 157-220

Oxford, UL, 120, s. 13-14, f. 1-70

Paris, Bibliothèque Mazarine, 742, s. 13, f. 172-?

desc. : http://www.calames.abes.fr/pub/\#details?id=MAZA13261

Paris, BNF, lat. 3630, s. 14, f. 75-96

repr. : https://gallica.bnf.fr/ark:/12148/btv1b100329427,

desc. : https://archivesetmanuscrits.bnf.fr/ark:/12148/cc61573x 
Paris, BNF, lat. 6838B, s. 13, f. 1-40v

repr. : https://gallica.bnf.fr/ark:/12148/btv1b90808394/f1.image

desc. : https://archivesetmanuscrits.bnf.fr/ark:/12148/cc65980n

Paris, BNF, lat. 11207, s. 13, f. 1-40,

repr. : https://gallica.bnf.fr/ark:/12148/btv1b53188245c,

desc. : https://archivesetmanuscrits.bnf.fr/ark:/12148/cc72823n

Paris, BNF, lat. 14297, s. 13, f. 148-168v

repr. : https://gallica.bnf.fr/ark:/12148/btv1b90777729

desc. : https://archivesetmanuscrits.bnf.fr/ark:/12148/cc75067g

Rouen, BM, 1468 (U 136), s. 13, f. 137-157v

Saint-Petersbourg, BNR, Lat. Qu.v.15, s. 15

Tübingen, UB, Mc 365, s. 13, f. 1-30

repr. : http://idb.ub.uni-tuebingen.de/opendigi/Mc365\#p=5\&tab=struct, incomplet

Wormsley, Paul Getty Jr's Library, 3731, s. 14 (ex.), f. 1-50

Wormsley, Paul Getty Jr's Library, 3747, s. 13, f. 1-29v

\section{Version $\mathrm{H}$}

Cambridge, Sidney Sussex College, 100, s. 13, f. 26-43

repr. partielle: http://iconographic.warburg.sas.ac.uk/vpc/VPC_search/

results_advanced_search.php? $\mathrm{p}=1 \&$ loc $=3464 \& \mathrm{msn}=873$

[*B-Hu2] Chalon-sur-Saone, BM, 14, s. 13, f. 55-89

repr. : https://bvmm.irht.cnrs.fr/resultRecherche/resultRecherche.php?

COMPOSITION_ID=7506

desc. : https://ccfr.bnf.fr/portailccfr/ark:/06871/004D12210042, feuillet sur le lion découpé : chapitre mutilé au début

Dresden, Sächs. Landesb., A.198, s. 13, f. 47-81

repr. : https://digital.slub-dresden.de/en/workview/dlf/217928/101/0/?

tx_dlf\%5Bpointer\%5D=0\&cHash=925b6f8a98d5f58d773d729ea391bd1e,

desc. : http://www.manuscripta-mediaevalia.de/?xdbdtdn!

\%22obj\%2040170840\%22\&dmode=doc\#\%7C4

Lüneburg, Ratsbücherei, Theol. 4o 20, s. 14

desc. : http://diglib.hab.de/?db=mss\&list=ms\&id=lg-rb-theol-4f-20\&catalog=Staehli

[ $\left.{ }^{*} \mathrm{~B}-\mathrm{Hu} 7\right]$ Paris, Bibliothèque Mazarine, 1029, s. 13, f. 87-96

desc. : http://www.calames.abes.fr/pub/\#details?id=MAZA14480

Paris, BNF, lat. 2495A, s. 12-13, desc. :

https://archivesetmanuscrits.bnf.fr/ark:/12148/cc60311q

Paris, BNF, lat. 2495B, s. 13, desc. :

https://archivesetmanuscrits.bnf.fr/ark:/12148/cc60312z

Paris, BNF, lat. 3638A, s. 13, f. 59v-93v

desc. : https://archivesetmanuscrits.bnf.fr/ark:/12148/cc61582w 
Paris, BNF, lat. 14429, s. 13, f. 109v-118

repr. : https://gallica.bnf.fr/ark:/12148/btv1b10033822c/f113.image

desc. : https://archivesetmanuscrits.bnf.fr/ark:/12148/cc751833

[*B-Hu3] Valenciennes, BM, 101, s. 13, f. 189-201

repr. partielle: https://bvmm.irht.cnrs.fr/resultRecherche/resultRecherche.php?

COMPOSITION_ID=6209

desc. : https://ccfr.bnf.fr/portailccfr/ark:/06871/004D30020249

Gdańs. Biblioteka Gdańska, 2224, s. 15, H/Seconde Famille?

Praha, Národní knihovna České republiky, V.A.7, s. 14, f. 35v-46, H/Seconde

Famille?

repr. : http://www.manuscriptorium.com/apps/index.php?direct=record\&pid=AIPDIG-

NKCR__V_A_7_-_-_-__28OZRUB-cs\#search

desc. : http://www.manuscriptorium.com/apps/index.php?direct=record\&pid=RASTISNKCR__V_A_7_-_-_-__280ZRUB-cs

Wroclaw, BU, I.F.281, s. 15, f. 350v-362, H/Seconde Famille?

\section{Famille dite de « Transition »}

[*B-Bes11] Cambridge, Gonville et Caius Coll., 384, s. 13, f. 167-199, longtemps donné comme appartenant à la Seconde famille, il s'agit plutôt d'un manuscrit atypique dont une partie correspond à la famille de Transition.

[ $\left.{ }^{*} B-T r 5\right]$ Cambridge, Trinity College, R.14.9 (884), s. 13, f. 89-106v

repr. : https://mss-cat.trin.cam.ac.uk/manuscripts/uv/view.php?n=R.14.9

desc. : https://mss-cat.trin.cam.ac.uk/viewpage.php?index=1159

London, BL, Cotton Vespasian E X, s. 13

London, BL, Royal 2 B VII, s. 14, f. 85-130v

repr. : http://www.bl.uk/manuscripts/Viewer.aspx?ref=royal_ms_2_b_vii_f084r desc. : http://www.bl.uk/manuscripts/FullDisplay.aspx?ref=Royal_MS_2_b_vii, psautier de la reine Marie. Pas de texte, uniquement programme iconographique.

[*B-Tr1] London, BL, Royal 12 C XIX, s. 12 (ex.), f. 1-94

repr. : http://www.bl.uk/manuscripts/Viewer.aspx?ref=royal_ms_12_c_xix_f006r desc. : http://www.bl.uk/manuscripts/FullDisplay.aspx?ref=Royal_MS_12_c_xix

[B-Tr4] Los Angeles, Getty Museum, 100, s. 13, f. 1-73, repr. partielle: http://www.getty.edu/art/collection/objects/240115/unknown-makernorthumberland-bestiary-english-about-1250-1260/

München, Bayerische Staatsbibliothek, gall. 16, s. 14

repr. : http://daten.digitale-sammlungen.de/bsb00056556/image_1

desc. : https://opacplus.bsb-muenchen.de/title/BV036859262, psautier de la reine Isabelle. Pas de texte, uniquement programme iconographique.

New York, Morgan Library, 81, s. 12 (ex.), f. 1-89

repr. partielle: http://ica.themorgan.org/manuscript/thumbs/77019

desc. : http://ica.themorgan.org/manuscript/description/77019

[B-Tr2] Saint-Petersbourg, BNR, Lat. Qu.v.V.1, s. 12 (ex.), f. 1-98 


\section{Troisième Famille}

[*B-Tert1] Cambridge, Fitzwilliam Museum, 254, s. 13 (inc.), f. 1-48

Cambridge, UL, Kk.4.25, s. 13, f. 55-100v

repr. : https://cudl.lib.cam.ac.uk/view/MS-KK-00004-00025/103

[*B-Tert2] London, Westminster Abbey, 22, s. 13, f. 1-54

Oxford, Bodl. Lib., Douce 88 E, s. 13 (ex.), f. 68-116v

repr. partielle: https://digital.bodleian.ox.ac.uk/inquire/p/

65563e6f-6e07-48e4-84da-40e7890de4d3

desc. : https://medieval.bodleian.ox.ac.uk/catalog/manuscript_4746

Oxford, Bodl. Lib., e Museo 136, s. 13, f. 1-47

repr. partielle: https://digital.bodleian.ox.ac.uk/inquire/p/4aa3ffc5-889d-4d9b-

a8d0-9fd0a7b5de64

desc. : https://medieval.bodleian.ox.ac.uk/catalog/manuscript_9087

\section{Dicta Chrysostomi}

Bad Windsheim, Ratsbibliothek, 28, s. 14

Bruxelles, Bibliothèque Royale, 18421-29, s. 13, f. 63-70v

Chicago, Newberry Library, 31.1, s. 14, f. 44-52v

desc. : https://webvoyage.carli.illinois.edu/nby/cgi-bin/Pwebrecon.cgi?

$\mathrm{DB}=$ local\&v1=1\&BBRecID=302562, olim Admont, Benediktinerstift, Cod. 483

[*DC4] Epinal, BM, 58 (209), s. 12, f. 70v-75

desc. : https://ccfr.bnf.fr/portailccfr/ark:/06871/004D03020152

Göttweig, Benediktinerstift, 154, s. 14, f. 105-114v

desc. : https://manuscripta.at/hs_detail.php?ID=36865

Göttweig, Benediktinerstift, 200, s. 15, f. 55r-72r

desc. : https://manuscripta.at/hs_detail.php?ID=36903

Harvard University, Houghton Library, Typ 101, s. 13, f. 60-89

Leipzig, UB, 351, s. 13, f. 128-133v

repr. : https://digital.ub.uni-leipzig.de/mirador/index.php\#0d1adda4-27fd-4ccb-805ed35ade274f 42

desc. : http://www.manuscripta-mediaevalia.de/obj31562770.html

Leipzig, UB, 1305, s. 15, f. 54v-63v

repr. : https://digital.ub.uni-leipzig.de/mirador/index.php\#070c9c87-

cd7a-416b-87a1-9ad18e011421

desc : http://www.manuscripta-mediaevalia.de/dokumente/html/obj90679971,T

Linz, Oberösterreichische Landesbibliothek, 33, s. 12-13, f. 139-171

repr. : https://digi.landesbibliothek.at/viewer/image/33/279/

desc. : https://digi.landesbibliothek.at/viewer/!metadata/33/279/-/ 
London, BL, Sloane 278, s. 13, f. 44-57

desc. : https://www.bl.uk/catalogues/illuminatedmanuscripts/record.asp?MSID=6512

München, Bayerische Staatsbibliothek, Clm 2655, s. 13 (ex), f. 95-105v

repr. : http://daten.digitale-sammlungen.de/bsb00112027/image_197

desc. : https://opacplus.bsb-muenchen.de/title/BV037448956

München, Bayerische Staatsbibliothek, Clm 3221, s. 14

[ $\left.{ }^{*} D C 5\right]$ München, Bayerische Staatsbibliothek, Clm 536, s. 12, f. 66

repr. : http://daten.digitale-sammlungen.de/bsb00007208/image_135

desc. : https://opacplus.bsb-muenchen.de/title/BV022217701

München, Bayerische Staatsbibliothek, Clm 5613, s. 15, f. 180-183v

desc. : https://opacplus.bsb-muenchen.de/title/BV037450643

München, Bayerische Staatsbibliothek, Clm 5921, s. 15, f. 282-292vv

desc. : https://opacplus.bsb-muenchen.de/title/BV037450828

[ $\left.{ }^{*} D C 9\right]$ München, Bayerische Staatsbibliothek, Clm 6908, s. 14, f. 78-85v

repr. : http://daten.digitale-sammlungen.de/ db/0007/bsb00078560/images.

desc. : https://opacplus.bsb-muenchen.de/title/BV037451231

München, Bayerische Staatsbibliothek, Clm 9600, s. 14, f. 127-134v

desc. : https://opacplus.bsb-muenchen.de/title/BV037452901

München, Bayerische Staatsbibliothek, Clm 14216, s. 15, f. 13v-17v, DC- ?

repr. : http://daten.digitale-sammlungen.de/bsb00036075/image_32

desc. : https://opacplus.bsb-muenchen.de/title/BV035381668

[*DC11] München, Bayerische Staatsbibliothek, Clm 14348, s. 13, f. 246-253v

repr. : http://daten.digitale-sammlungen.de/bsb00046577/image_495

desc. : https://opacplus.bsb-muenchen.de/title/BV036063271

München, Bayerische Staatsbibliothek, Clm 14693, s. 11-12, f. 111-116v

repr. : http://daten.digitale-sammlungen.de/bsb00093773/image_224

desc. : https://opacplus.bsb-muenchen.de/title/BV021530096

München, Bayerische Staatsbibliothek, Clm 16189, s. 15, f. 100-109v

desc. : https://opacplus.bsb-muenchen.de/title/BV036862277

München, Bayerische Staatsbibliothek, Clm 19648, s. 15, f. 180-194v

desc. : https://opacplus.bsb-muenchen.de/title/BV037456650

München, Bayerische Staatsbibliothek, Clm 23787, s. 15, f. 12-19v

desc. : https://opacplus.bsb-muenchen.de/title/BV037457838

[*DC1] New York, Morgan Library, 832, s. 11

repr. : http://ica.themorgan.org/manuscript/thumbs/158985

desc. : http://ica.themorgan.org/manuscript/description/158985, olim Göttweig

Oxford, Saint John's College, 136, s. 13

Paris, Bibliothèque de l'Arsenal, Ms-394, s. 13, f. 172-183

desc. : https://archivesetmanuscrits.bnf.fr/ark:/12148/cc795773 
Paris, BNF, lat. 2780, s. 12 (ex.), f. 93-115v

repr. : https://gallica.bnf.fr/ark:/12148/btv1b100334523

desc. : https://archivesetmanuscrits.bnf.fr/ark:/12148/cc60563k

Paris, BNF, lat. 2843E, s. 13 (ex.), f. 64-79v, DC?

desc. : https://archivesetmanuscrits.bnf.fr/ark:/12148/cc60639k

Paris, BNF, lat. 10448, s. 13, f. 118v

repr. : http://gallica.bnf.fr/ark:/12148/btv1b9066069t

desc. : https://archivesetmanuscrits.bnf.fr/ark:/12148/cc72169q

Saint-Petersbourg, BNR, Lat. Qu.v.III.3, s. 14, f. 37

Wien, ONB, lat. 303, s. 13 (ex.), f. 124v-130v, DC/Theobaldi

desc. : http://data.onb.ac.at/rec/AC14392857

[ $\left.{ }^{*} D C 3\right]$ Wien, ONB, lat. 1010, s. 12, f. 65-73

desc. : http://data.onb.ac.at/rec/AC13958584

Wien, ONB, lat. 2511, s. 14, f. 135r-140v

desc. : http://data.onb.ac.at/rec/AC13976466

Wien, ONB, lat. 4609, s. 12, f. 65-73

desc. : http://data.onb.ac.at/rec/AC13958584

Wien, ONB, lat. 13378, s. 13, f. 1-7v

desc. : http://data.onb.ac.at/rec/AC13968683

Wolfenbüttel, Herzog August Bibliothek, Cod. Guelf. 35a Helmst., s. 14, f. 1-1v repr. : http://diglib.hab.de/mss/35a-helmst/start.htm

desc. : http://diglib.hab.de/?db=mss\&list=ms\&id=35a-helmst\&catalog=Heitzmann, fragmentaire et très abimé

\section{Version non identifiée à ce jour}

$\left[{ }^{*} A v\right]$ Avranches, BM, 28, s. 13, f. 179-180

repr. : https://bvmm.irht.cnrs.fr/consult/consult.php?reproductionId=871

desc. : http://www.unicaen.fr/bvmsm/ead.html?id=FR_UCBN_MSM_mss_av

Cambridge, Corpus Christi College, 448, s. 10, f. 88-89v

repr. : https://parker.stanford.edu/parker/catalog/kk233ff6415, ne contient que lion, licorne, panthère

Chartres, BM, 63 (125), s. 10-11, f. 67v-70

desc. : https://ccfr.bnf.fr/portailccfr/ark:/06871/004D17010314, manuscrit détruit

Città del Vaticano, BAV, Pal. lat. 1064, s. 13, f. 35v-48

repr. : https://digi.vatlib.it/view/MSS_Pal.lat.1064

Città del Vaticano, BAV, Reg. lat. 258, s. 13 (inc.), f. 8-42v

repr. : https://digi.vatlib.it/view/MSS_Reg.lat.258

London, BL, Add. 22041, s. 13, extraits

London, BL, Add. 24097, s. 13 
London, BL, Royal 10 A VII, s. 13, f. 215-216

desc. : https://www.bl.uk/catalogues/illuminatedmanuscripts/record.asp?

MSID=5358\&ColliD=16\&NStart=100107, extraits d'un bestiaire moralisé

$\left[{ }^{*} R\right]$ London, BL, Royal 6 A XI, s. 12, f. 141-146

desc. : http://www.bl.uk/catalogues/illuminatedmanuscripts/record.asp?MSID=6044

$\left[{ }^{*} L\right]$ Lyon, BM, 125, s. 15, f. 189-196v

desc. : https://ccfr.bnf.fr/portailccfr/ark:/06871/004D33A10227

Oxford, Bodl. Lib., Lat. th.e.9, s. 12, f. 57-58

desc. : https://medieval.bodleian.ox.ac.uk/catalog/manuscript_6805, extraits

\section{Annexe 2 : Tables des matières comparées des principaux manuscrits dans toutes les versions}

Le document est proposé en téléchargement ici en deux formats :

Tables des matières comparées (couleurs, A3) : cf. document joint ci-dessous.

Tables des matières comparées (noir et blanc, A3) : cf. document joint ci-dessous.

\section{Annexe 3 : Codes des zoonymes}

Liste des sigles utilisés dans le document « Tables des matières comparées » : cf. document joint ci-dessous.

\section{NOTES}

1. Sur le Physiologos grec, on pourra consulter : SBORDONE F., Ricerche sulle fonti e sulla composizione del Physiologus greco, Napoli, 1936 ; LAZARIS S., Le Physiologus grec, I: la réécriture de l'histoire naturelle antique, Firenze, 2016. Le texte est traduit dans: ZUCKER A., Physiologos: le bestiaire des bestiaires, Grenoble, 2004.

2. Sur les éléments de datation de la première traduction latine, voir : HENKEL N., Studien zum Physiologus im Mittelalter, Tübingen, 1976, p. 23 ; ORLANDI G., "La tradizione del Physiologus e i prodromi nel bestiaro latino ", in L'uomo di fronte al mondo animale nell'alto Medioevo, Spolète, 1985, p. 1057-1106, ici p. 1067-1068 ; KUHRY E., «Les chapitres sur les poissons et créatures aquatiques dans la tradition manuscrite du Physiologus latinus et des bestiaires latins et leur réception dans quelques encyclopédies médiévales ", in Inter litteras et scientias. Recueil d'études en hommage à Catherine Jacquemard, AVENEL, M.-A. - GAUVIN, B., (éd.), Caen, 2019, p. 117-149, ici p. 117.

3. On trouvera une bibliographie détaillée sur le Physiologus dans les autres langues dans : PAKIS V. A., «Contextual Duplicity and Textual Variation: The Siren and Onocentaur in the Physiologus Tradition ", Mediaevistik, 23, 2010, p. 115-85.

4. Sur les différentes familles issues de B identifiées par M. R. James et précisées par F. McCulloch, on consultera JAMES M. R., The Bestiary, Being a Reproduction in full of the Ms. Ii.4.26 in the University Library, Oxford, 1928 ; MCCULLOCH F., Mediaeval Latin and French bestiaries, Chapel Hill, 1962 ; STEWART P., The medieval bestiary and its textual tradition, Thèse de l'University of St. Andrews, 2012 ; KAY S., « The English Bestiary, the Continental Physiologus, and the Intersections between Them », Medium Aevum, 85 (1), 2016, p. 118-42. S. Kay en particulier fait une intéressante 
analyse de la dichotomie entre les travaux anglo-saxons, fidèles aux familles définies par M. R. James et centrés sur le bestiaire (considéré comme nativement anglais), souvent dans une perspective d'histoire de l'art, et les travaux continentaux (allemands et italiens pour la plupart), centrés sur le Physiologus ancien et les versions ultérieures continentales comme les Dicta Chrysostomi et le Physiologus Theobaldi.

5. Le premier livre du pseudo-De Bestiis est le De avibus d'Hugues de Fouilloy, le troisième un bestiaire de Seconde Famille, et le quatrième le recueil de propriétés Angelus purus natura : CLARK W. B., "Four Latin bestiaries and De bestiis et aliis rebus", in Bestiaires médiévaux. Nouvelles perspectives sur les manuscrits et les traditions textuelles, VAN DEN ABEELE, B., (éd.), Louvain-la-Neuve, 2005, p. 49-69 ; KUHRY E., « Dictionnaires, distinctions, recueils de propriétés en milieu cistercien : outils pour la prédication, sources pour l'étude de la nature ", in Les Cisterciens et la transmission des textes (XII ${ }^{e}$-XVIII ${ }^{e}$ siècles), TURCAN-VERKERK, A.-M., FALMAGNE, Th., STUTZMANN, D., et GANDIL, P. (éd.), Turnhout, 2018, (Bibliothèque d'histoire culturelle du Moyen Âge, 18), p. 285-337.

6. Je remercie très sincèrement $A$. Zucker, pour l'environnement de travail propice que j'ai pu trouver lors de mon post-doctorat au CEPAM de Nice, Th. Buquet (CRAHAM, CNRS-Université de Caen Normandie) et A. Dorofeeva (Durham University), pour le signalement de plusieurs témoins qui m'étaient inconnus, C. Macé (Akademie der Wissenschaften zu Göttingen) pour avoir partagé sa propre liste de manuscrits. Enfin je remercie l'Institut de Recherche et d'Histoire des Textes en la personne de son directeur F. Bougard pour l'accès à la riche collection de microfilms du laboratoire qui a largement contribué à rendre cette étude possible.

\section{RÉSUMÉS}

Cet article propose une mise au point sur la tradition manuscrite du Physiologus latin et des bestiaires dérivés sous la forme d'une rapide introduction et d'un ensemble de trois ressources pour son étude: une liste des manuscrits classés par version (153 entrées); les tables des matières comparées des principaux manuscrits dans toutes les versions; une liste des sigles utilisés dans les tables pour représenter les zoonymes, noms de pierres et noms de plantes.

This contribution aims at an update on the subject of the manuscript tradition of the Latin Physiologus and of the medieval bestiary in the form of a short introduction and three resources for the Physiologus' study: a list of witnesses ordered by version (153 items); the tables of contents of the main manuscripts in each version; a list of the sigla used in the tables to represent zoonyms, names of stones and of plants.

\section{INDEX}

Mots-clés : Physiologus Latinus, bestiaire, manuscrits

Keywords : Physiologus Latinus, Bestiary, Manuscripts 


\section{AUTEUR}

\section{EMMANUELLE KUHRY}

Emmanuelle Kuhry est post-doctorante à l'Institut de Recherche et d'Histoire des Textes (UPR 841 - CNRS). Ses recherches portent sur la transmission manuscrite de la philosophie naturelle. Son projet actuel vise à l'étude et l'édition électronique d'un corpus de gloses universitaires de la première moitié $\mathrm{du} \mathrm{XIII}^{\mathrm{e}}$ siècle aux traités d'Aristote sur la nature. Elle a réalisé en 2016 un postdoctorat au CEPAM de Nice sur la tradition manuscrite du Physiologus latin. 